\title{
Comparative in vitro activities of eight antifungal drugs against a national collection of clinical Candida parapsilosis complex
}

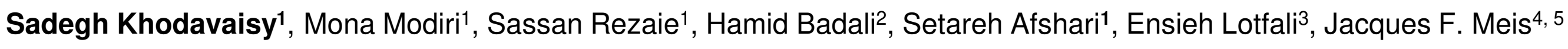

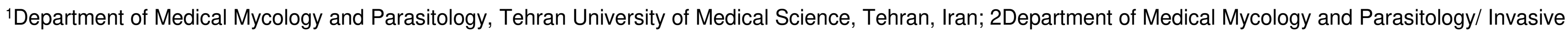

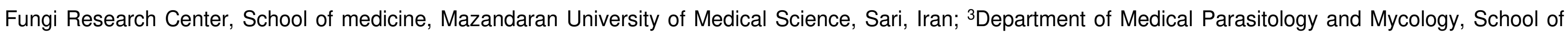

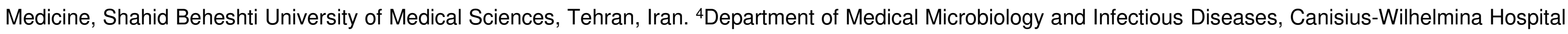
(CWZ), Nijmegen, The Netherlands, ${ }^{5}$ Centre of Expertise in Mycology Radboudumc/CWZ, Nijmegen, The Netherlands

Candida parapsilosis complex is composed of three distinct species, namely C. parapsilosis sensu stricto, C. orthopsilosis, and $C$. metapsilosis. This species complex is a significant cause of bloodstream infections in otherwise healthy individuals. Although $C$. parapsilosis complex is usually susceptible to most antifungal agents, recent studies have reported decreased susceptibility to azoles and echinocandins, as well as frequent relapses and failures. The current study aimed to determine the in vitro activities of echinocandins (i.e., anidulafungin and micafungin) and five comparator drugs against a large national collection of clinical $C$. parapsilosis complex obtained from different geographical regions of Iran, where $C$. parapsilosis is one of the common non-albicans Candida species.
The collection consisted of 87 clinical strains of $C$. parapsilosis complex isolates obtained from a variety of specimens, including nail $(n=63)$, skin lesions $(n=12)$, groin $(n=6)$, ear swabs $(n=2)$ blood $(n=2)$, vaginal discharge $(n=1)$, and sputum $(n=1)$. All isolates were initially identified to species level by DNA sequencing of ITS rDNA region. They were also reconfirmed using the matrix-assisted laser desorption ionization-time of flight mass spectrometry. In vitro antifungal susceptibility testing was adjusted according to Clinical and Laboratory Standards Institute (CLSI) guidelines.

In vitro susceptibilities of antifungal drugs against clinical isolates of $C$. parapsilosis complex.

\begin{tabular}{|c|c|c|c|c|c|c|c|c|c|c|c|c|c|c|c|c|c|}
\hline \multirow{2}{*}{$\begin{array}{l}\text { Strains and } \\
\text { antifungal } \\
\text { drugs }\end{array}$} & \multicolumn{17}{|c|}{ MICs ( $\mu \mathrm{g} / \mathrm{ml})$} \\
\hline & Range & MIC50/MIC90 & GM & $\% \leq \mathrm{ECV}^{*}$ & 0.008 & 0.016 & 0.031 & 0.063 & 0.125 & 0.25 & 0.5 & 1 & 2 & 4 & 8 & 16 & $>32$ \\
\hline \multicolumn{18}{|c|}{ All C. parapsilosis isolates $(n=87)$} \\
\hline AMB & $0.016-2$ & $0.016 / 0.15$ & 0.0314 & 100 & & 58 & 7 & 11 & 3 & 2 & & 2 & 4 & & & & \\
\hline FLU & $0.25-8$ & $1 / 2$ & 1.0573 & 93.1 & & & & & & 1 & 18 & 49 & 13 & 4 & 2 & & \\
\hline ITC & $0.031-2$ & $0.25 / 1$ & 0.211 & 88.5 & & & 2 & 21 & 20 & 11 & 23 & 6 & 4 & & & & \\
\hline VRC & $0.016-0.125$ & $0.016 / 0.031$ & 0.0176 & 100 & & 76 & 10 & & 1 & & & & & & & & \\
\hline POS & $0.016-0.125$ & $0.016 / 0.016$ & 0.0101 & 100 & & 81 & 2 & 3 & 1 & & & & & & & & \\
\hline AFG & $0.008-1$ & $0.25 / 0.5$ & 0.2081 & 100 & 2 & 1 & 1 & 7 & 18 & 36 & 18 & 4 & & & & & \\
\hline MFG & $0.008-1$ & $0.125 / 0.31$ & 0.1268 & 100 & 3 & & 10 & 15 & 19 & 32 & 7 & 1 & & & & & \\
\hline \multicolumn{18}{|c|}{ C. parapsilosis sensu stricto $(n=75)$} \\
\hline AMB & $0.016-2$ & $0.016 / 0.125$ & 0.0308 & 100 & & 53 & 5 & 7 & 3 & 1 & & 2 & 4 & & & & \\
\hline FLU & $0.25-8$ & $1 / 2$ & 0.9726 & 93.3 & & & & & & 1 & 18 & 46 & 5 & 3 & 2 & & \\
\hline ITC & $0.031-2$ & $0.125 / 0.5$ & 0.1821 & 92 & & & 2 & 21 & 19 & 8 & 19 & 4 & 2 & & & & \\
\hline VRC & $0.016-0.125$ & $0.016 / 0.016$ & 0.0171 & 100 & & 69 & 5 & & 1 & & & & & & & & \\
\hline POS & $0.016-0.031$ & $0.016 / 0.016$ & 0.0110 & 100 & & 71 & 3 & 1 & & & & & & & & & \\
\hline AFG & $0.008-1$ & $0.25 / 0.5$ & 0.2137 & 100 & 2 & 1 & 1 & 5 & 15 & 30 & 17 & 4 & & & & & \\
\hline MFG & $0.008-1$ & $0.25 / 0.5$ & 0.1461 & 100 & 3 & & 4 & 11 & 18 & 31 & 7 & 1 & & & & & \\
\hline \multicolumn{18}{|c|}{ C. orthopsilosis $(\mathrm{n}=12)$} \\
\hline AMB & $0.016-0.25$ & $0.031 / 0.125$ & 0.0352 & 100 & & 5 & 2 & 4 & & 1 & & & & & & & \\
\hline FLU & $1-4$ & $2 / 4$ & 1.7817 & 91.6 & & & & & & & & 3 & 8 & 1 & & & \\
\hline ITC & $0.125-2$ & $0.5 / 2$ & 0.5297 & 66.6 & & & & & 1 & 3 & 4 & 2 & 2 & & & & \\
\hline VRC & $0.016-0.031$ & $0.016 / 0.031$ & 0.0210 & 100 & & 7 & 5 & & & & & & & & & & \\
\hline POS & $0.016-0.016$ & $0.016 / 0.016$ & 0.0170 & 100 & & 10 & 2 & & & & & & & & & & \\
\hline AFG & $0.062-0.5$ & $0.25 / 0.5$ & 0.1765 & 100 & & & & 2 & 3 & 6 & 1 & & & & & & \\
\hline MFG & $0.031-0.25$ & $0.031 / 0.125$ & 0.0522 & 100 & & & 6 & 4 & 1 & 1 & & & & & & & \\
\hline
\end{tabular}

All strains had low MICs for posaconazole, voriconazole, itraconazole, followed by anidulafungin and micafungin. Fluconazole was found to be the less active drugs. The GM MIC values of posaconazole and voriconazole against all $\mathrm{C}$. parapsilosis complex isolates were $>6$-fold and > 5-dilution steps lower than those of fluconazole, respectively. $56 \%$ and $8 \%$ of $C$. parapsilosis sensu stricto isolates were susceptible and resistant to itraconazole, respectively. 27 (36\%) isolates were susceptible-dose dependent, one of which was also susceptibledose dependent to fluconazole. On the contrary, 1 (8.34\%), 7 $(58.33 \%)$, and $4(33.33 \%)$ isolates of $C$. orthopsilosis were susceptible, susceptible-dose dependent, and resistant to itraconazole, respectively. However, no statistically significant difference was observed among the $C$. parapsilosis sensu stricto and $C$. orthopsilosis isolates in terms of the MIC values for posaconazole, voriconazole and anidulafungin $(P>0.05)$.
Distribution of $C$. parapsilosis complex species within different age groups, gender and sites of isolation

\begin{tabular}{|c|c|c|c|c|}
\hline & & $\begin{array}{c}\text { C. parapsilosis } \\
\text { sensu stricto } \\
(n=75)\end{array}$ & $\begin{array}{l}\text { C. orthopsilosis } \\
\qquad(n=12)\end{array}$ & $\begin{array}{l}\text { Total } \\
(n=87)\end{array}$ \\
\hline \multirow[t]{3}{*}{ Gender } & Male & 27 (36\%) & $3(25 \%)$ & $30(34.5 \%)$ \\
\hline & Female & $48(64 \%)$ & $9(75 \%)$ & $57(65.5 \%)$ \\
\hline & $0-15$ & $6(8 \%)$ & 0 & $6(6.9 \%)$ \\
\hline \multirow{3}{*}{ Age (years) } & $16-29$ & $15(20 \%)$ & $3(25 \%)$ & $18(20.68 \%)$ \\
\hline & $30-49$ & $30(40 \%)$ & $3(25 \%)$ & $33(37.94 \%)$ \\
\hline & $\geq 50$ & $24(32 \%)$ & $6(50 \%)$ & $30(34.48 \%)$ \\
\hline \multirow{7}{*}{$\begin{array}{l}\text { Site of } \\
\text { isolation }\end{array}$} & Groin & $5(6.6 \%)$ & $1(8.3 \%)$ & $6(6.9 \%)$ \\
\hline & Nail & $53(70.6 \%)$ & $10(83.3 \%)$ & $63(72.41 \%)$ \\
\hline & Skin & $11(14.6 \%)$ & $1(8.3 \%)$ & $12(13.7 \%)$ \\
\hline & Sputum & $1(1.3 \%)$ & 0 & $1(1.15 \%)$ \\
\hline & Ear & $2(2.6 \%)$ & 0 & $2(2.3 \%)$ \\
\hline & Vaginitis & $1(1.3 \%)$ & 0 & $1(1.15 \%)$ \\
\hline & Blood & $2(2.6 \%)$ & 0 & $2(2.3 \%)$ \\
\hline
\end{tabular}

